\title{
Learning from peer review
}

\author{
Encouraging PhD students to engage with the peer-review process is of benefit to researchers, journals \\ and the rest of the scientific community, says Bryden Le Bailly.
}

$\mathrm{P}$ eer review is a key pre-publication step in the reporting of research. At its best, this process critically evaluates all aspects of a submitted article, ensuring that the papers that are eventually published meet the journal's criteria while maintaining a high level of scientific practice. The review process also plays another important role: it provides an evaluation of the authors' work from an external source. Both reading and writing this feedback can be incredibly useful for training researchers, giving them an appreciation of how criticism is fundamental to improving their work and the way in which it is presented.

Unfortunately, most postgraduates only fleetingly come into contact with peer review during their studies. A simple way of improving this situation is for the students themselves to referee manuscripts. This approach could prove a significant tool in training the next generation of researchers. Moreover, this largely untapped resource can be used to great advantage not only for the students, but for academics, journals and the wider scientific community.

I started peer reviewing papers, in tandem with my supervisor, at the beginning of my postdoctoral position. But given the chance, I wish I'd been involved earlier. My PhD project involved learning a lot of new information from a whole range of fields: organometallic chemistry, chemical biology, supramolecular chemistry - the list goes on. Reading submitted manuscripts has meant that I approach published articles differently. Rather than idly browsing the text and figures, I tackle the task more purposefully, quickly disseminating the key information: impact, novelty, limitations. Moreover, it has indirectly improved my own research.

As many people, I work in a research group with more than one area of interest (in my case, organolithium rearrangment chemistry and molecular communication devices) so need to have a good breadth of knowledge to help students with their day-to-day issues. Critically evaluating manuscripts has led me to broaden my general knowledge by reading similar, but not directly related, chemistry to my research. This has given me a greater appreciation of where my work sits in the broader literature. In fact, reviewing one paper led me to find inspiration from one of its references. Reading this seemingly unrelated science eventually led to making a breakthrough in something I'd been trying to get to work in the lab for months, just by thinking about it differently. Eventually this led me to attend a conference in India well outside my field, learning about chemistry I had no real knowledge of before.

\section{Given the proper guidance, PhD students can help expand and even improve peer review. Not only will this advance their written and communication skills, it can give them insight into the publication process and indirectly improve their \\ own research.}

Reviewing papers also helps improve important communication skills that are, especially in a lab environment, often left in second place to the practical side of things. It is important to come into contact with both well and badly written science to understand how to convey your work. Already in my limited experience I have seen a number of manuscripts where the scientific content was effectively hidden behind overly complicated prose and a poor use of English. Peer review is a good way of considering the presentation of your own work, which will come in handy at conferences, in writing theses and in preparing papers for publication.

To expand the refereeing pool with students, more academics need to engage in training the next generation of reviewers. This will significantly reduce the burden of peer review on the current refereeing pool who are limited in the time they can provide for assessing submitted manuscripts. Supervisors should also see this as a long-term gain as part of graduate training. Clearly, this may not be suitable for all students, but encouraging even a handful of interested postgraduates in each research group would benefit both them and their often overstretched supervisors. An alternative idea would be to democratize the process. Many groups already present published literature in their regular meetings, so why not start to referee papers together as well? In theory this approach should enhance the quality and quantity of feedback for a given paper. Necessarily the quality of the articles published and the confidentiality of the process need to be maintained, but both of these approaches, when done in concert with a supervisor, will significantly give students a set of skills that is difficult to obtain from research alone. And for reluctant participants, reviewing papers is a good skill to have on your CV - it shows initiative, commitment outside of the 'required' parameters of a $\mathrm{PhD}$, and will be useful in the long term, whether in academia or elsewhere.

While the reviewing of papers is integral to scientific communication, it is also one of the slowest steps in the publication process. As the rate of research quickens, we need methods to keep up with the increasing pace without reducing the quality of refereeing. Expanding the referee pool with $\mathrm{PhD}$ students is a simple way to do this. For better or worse, we work in a system where publication records determine careers, so it's in all our interests to ensure that the review process is as comprehensive and efficient as possible. Given the proper guidance, $\mathrm{PhD}$ students can help expand and even improve peer review. Not only will this advance their written and communication skills, it can give them insight into the publication process and indirectly improve their own research. If it is done in the right way, we will all reap the benefits.
BRYDEN LE BAILLY is a postdoctoral researcher working on conformational signal relay with dynamic foldamers at the School of Chemistry, University of Bristol, Cantock's Close, Bristol BS8 1TS, UK. e-mail: bryden.lebailly@bristol.ac.uk 